\title{
Ultrasonic Liver Image Denoising Based on A Hybrid Threshold Method
}

\author{
H.J. Zhu \\ College of Information Science \& Technology \\ Beijing University of Chemical Technology \\ Beijing, China, 100029
}

\author{
L. Rao \\ Jiangxi Normal University \\ Nanchang, China, 330027
}

\begin{abstract}
The objective of this paper is to investigate a hybrid threshold denoising algorithm based on wavelet transform for the ultrasonic liver image. A novel hybrid threshold function first is discussed. The hybrid threshold denoising algorithm based on the wavelet transform is then performed for ultrasound image of the liver. Only is one parameter selected in the proposed image denoising algorithm. Several metrics such as correlation coefficient (CoC), edge preservation index (EPI), and structural similarity index (SSI) are measured to quantify the denoised results of ultrasound liver image. Experiments show that the wavelet-based hybrid threshold denoising algorithm is effective and feasible.
\end{abstract}

Keywords-ultrasonic liver image; the hybrid threshold function; denoising; wavelet transform

\section{INTRODUCTION}

Ultrasound image plays an important role in the medical diagnosis and medical clinic. Unfortunately, some existential speckle noises and grey level discontinuity degrade the ultrasound images. It is difficult for the observer to identify clearly the interest details. Therefore, medical ultrasound image denoising is considered as one of the major problems in the medical ultrasonography.

Because speckle noise degrades the details and contrast resolution of medical ultrasound images, ultrasound image denoising is a key technique for medical diagnosis and medical clinic. In the reported literatures, speckle reduction approaches for medical ultrasound image include spatial filtered method $[1,3,5,8,9,11]$ and multiscale denoising methods $[2,4,6,7,10,14,16]$.

In the filtered methods some prominent edges of tissues or organs were preserved or enhanced after speckle noises removed. Koo [1] explored a homogeneous region growing mean filter for medical ultrasonic images speckle noising reduction. This method reduced speckle noise with edge preservation, but a proper seed region was difficult to determine at a larger speckle noise size. Damodaran [9] presented a discrete topological derivative image denoising approach to improve hyperechoic regions resulting in ultrasound medical images diagnosis. It is difficult to obtain good effect in the weak hyperechoic regions of ultrasound medical images.

Multiscale denoising methods have attracted more and more researchers' attention due to their excellent localization property.
Harmoko [14] combined a wavelet multi-scale strategy and a warping optical flow to generate a high-accuracy velocity vector from two consecutive frames of poor-quality ultrasound images. Gupta [16] provided a generalized Nakagami probability density function to denoise the medical ultrasound images. Essentially, most of these reported literatures using wavelet strategy needed to adjust multiple parameters to denoise the ultrasound images.

In medical ultrasound image, the abdominal or the liver ultrasound is one of the common ways to diagnose liver disorders and diseases. The objective of this paper is to investigate the hybrid threshold denoising algorithm based on the wavelet transform for the ultrasonic image of the liver. The proposed method constructs a hybrid threshold function with one selected parameter.

\section{PROPOSED APPROACH}

It is well known that the hard function is discontinuity and the soft function has bigger bias in Donoho's algorithm [12]. Some improved methods $[7,13,15]$ were proposed in succession, but multiple parameters were tuned to denoise in these methods.

To reduce the drawbacks of thresholding function in Donoho's algorithm and the adjustment of more parameters in the thresholding function, we investigate a hybrid threshold function. The hybrid threshold function is constructed as

$$
\hat{X}=\left\{\begin{array}{cl}
\operatorname{sgn}(X)\left(|X|-\frac{T}{\alpha^{-|X|-T}}\right) & |X|>T \\
0 & |X| \leq T
\end{array}\right.
$$

Where $X$ is the wavelet coefficients, $\hat{X}$ is the corrected wavelet coefficients, and $\alpha$ is one selected parameter. The hybrid threshold function only needs to adjust one free parameter.

An analytical express for the hybrid threshold function is discussed in the following. While the parameter $\alpha$ is very close to 0 , we can get

$$
\lim _{\alpha \rightarrow 0} \operatorname{sgn}(X)\left(|X|-\frac{T}{\alpha^{-|X|-T}}\right)=\operatorname{sgn}(X)|X|=\frac{X}{|X|} \cdot|X|=X
$$


The function Eq. 2 is approximate to the hard-threshold function. When the parameter $\alpha$ is very close to 1 , we have

$$
\lim _{\alpha \rightarrow 1} \operatorname{sgn}(X)\left(|X|-\frac{T}{\alpha^{-|X|-T}}\right)=\operatorname{sgn}(X)(|X|-T)
$$

The function Eq. 3 is close to the soft-threshold function. From Eq. 1, Eq. 2 and Eq. 3, we can see that the hybrid threshold function is a feasible threshold selection between the soft threshold and the hard threshold function. The parameter $\alpha \in(0,1]$ is selectable.

Assuming that a function $f(X)$ is equal to the first part in Eq. 1, that is

$$
f(X)=\operatorname{sgn}(X)\left(|X|-\frac{T}{\alpha^{-|X|-T}}\right)
$$

For $X<0$, we get $\lim _{X \rightarrow-\infty} \frac{f(x)}{X}=1$, while $X>0$, we $\lim _{\text {have }} \frac{f(X)}{X}=1$. And we have

$$
\lim _{X \rightarrow+\infty}(f(X)-X)=\lim _{X \rightarrow+\infty}\left(\operatorname{sgn}(X)\left(|X|-\frac{T}{\alpha^{-|X|-T}}\right)-X\right)=\lim _{X \rightarrow+\infty}\left(-\frac{T}{\alpha^{-X-T}}\right)=0
$$

Eq. 5 can be used to explain the hybrid threshold function approximated gradually the line $\hat{X}=X$. That is to say, this hybrid threshold function can reduce the bigger bias between the original wavelet coefficients $X$ and the estimated wavelet coefficients $\hat{X}$ in the soft- threshold function. Besides, the hybrid threshold function is continuous at $|X|=T$ and can decrease the bigger variance because of the discontinuity of the hard-threshold function.

The ultrasound image denoising algorithm based on the hybrid threshold function is briefly summarized as follows.

Step 1: Estimation of wavelet coefficients for original image.

Step 2: Computation of threshold by the hybrid threshold function $T=\sigma \sqrt{2 \log (M)}$.

Step 3: Computing the parameter $\alpha$ from Eq. 6 .

$$
\alpha=E_{I_{s}} / E_{I_{o}}=\sum_{i=0}^{W-1} \sum_{j=0}^{H-1} I_{s}{ }^{2}(\mathrm{i}, j) / \sum_{i=0}^{W-1} \sum_{j=0}^{H-1} I_{o}{ }^{2}(\mathrm{i}, j)
$$

Where ${ }^{I_{o}}$ be an original image and ${ }^{I_{s}}$ represent the smoothed result using weighted median filter.
Step 4: Denoising the original image through estimating the corrected wavelet coefficients from Eq. 1 and the computed parameter $\alpha$ in Step 3.

To evaluate the proposed method, several metrics such as correlation coefficient (CoC) [4], edge preservation index (EPI) [1,4], and structural similarity index (SSI) [4] were calculated from the diagnosed results. Correlation coefficient and structural similarity index are measured of similarity between the original and denoised images, and EPI denotes to restore or preserve the edges after a speckle reduction method. These quality metrics defined as follows:

$$
\begin{gathered}
C o C=\frac{\sum\left(I_{o}-\bar{I}_{o}\right)\left(I_{d}-\bar{I}_{d}\right)}{\sqrt{\sum\left(I_{o}-\bar{I}_{o}\right)^{2} \sum\left(I_{d}-\bar{I}_{d}\right)^{2}}}, \\
E P I=\frac{\sum\left(\Delta I_{o}-\Delta \bar{I}_{o}\right)\left(\Delta I_{d}-\Delta \bar{I}_{d}\right)}{\sqrt{\sum\left(\Delta I_{o}-\Delta \bar{I}_{o}\right)^{2} \sum \Delta\left(I_{d}-\Delta \bar{I}_{d}\right)^{2}}}, \\
S S I=\frac{\left(2 \bar{I}_{o} \bar{I}_{d}+C_{1}\right)\left(2 \sigma_{I_{o} I_{d}}+C_{2}\right)}{\left(\bar{I}_{o}^{2}+\bar{I}_{d}^{2}+C_{1}\right)\left(\sigma_{I_{o}}^{2}+\sigma_{I_{d}}^{2}+C_{2}\right)}
\end{gathered}
$$

Where $I_{o}$ is original image, $I_{d}$ is denoised image, ${ }^{I_{o}}$ shows the mean of $I_{o}, \bar{I}_{d}$ shows the mean of $I_{d}, \Delta\left(I_{o}\right)$ is the high-pass filtered of ${ }^{I_{o}}$ using the discrete Laplacian operator, and $\sigma_{I_{o}}^{2}$ the variance of $I_{o}, \sigma_{I_{d}}^{2}$ the variance of $I_{d}, \sigma_{I_{o} I_{d}}$ the covariance of $I_{o}$ and $I_{d}, C_{1}=\left(k_{1} L\right)^{2}$ and $C_{2}=\left(k_{2} L\right)^{2}$ two variables to stabilize the division with weak denominator, $L=225$ the dynamic range of the pixel values, $k_{1}=0.01$ and $k_{2}=0.03$.

\section{EXPERIMENTAL RESULTS AND DISCUSSION}

The first experiment tested the influence of the parameter $\alpha$ on the proposed method. One real ultrasound image corrupted by speckle noise with variance $\sigma=0.01$, $\sigma=0.05, \sigma=0.3$ and $\sigma=0.5$ was first generated. The proposed method was then applied to eliminate the speckle noise of the corrupted ultrasound image. The experimental results are shown in Figure 1.The peak signal to noise ratio (PSNR) of the denoised results and the parameters were shown in Table 1.

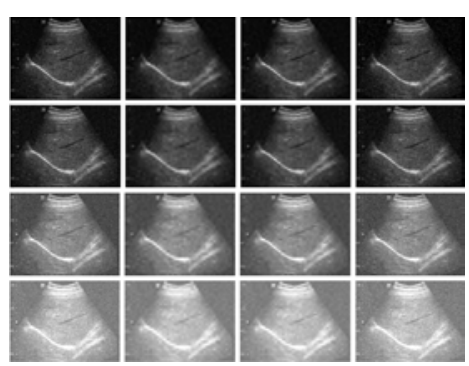

FIGURE I. FROM LEFT TO RIGHT: THE CORRUPTED ULTRASOUND IMAGE; DENOISING WITH THE SOFT-THRESHOLD FUNCTION, DENOISING WITH THE HARD-THRESHOLD FUNCTION; AND DENOISING WITH THE PROPOSED METHOD. 
In the second experiment, the parameter $\alpha$ is first estimated for three real ultrasound liver images, and these images are denoised using the proposed method and Damodaran's method [9]. The experimental results are shown in Figure 2. For the three ultrasound liver images, the parameter $\alpha$ is $0.9984,0.9742$ and 0.9854 , respectively. We can see that the denoised effect of the proposed method is better than that of Damodaran's method [9] in Figure 2.

Here, we use COC, EPI, and SSI quality metrics to better evaluate the proposed denoising algorithm. Table 2 shows these metrics of the second image in Figure 2 which is added speckle noise with variance $\sigma=0.1$ and $\sigma=0.5$. From the result, it can be seen that the key parameter $\alpha$ plays an important role in the proposed wavelet-based hybrid thresholding method.
TABLE I. THE ESTIMATED PARAMETERS $\alpha$ AND PSNRS OF THE DENOISED RESULTS.

\begin{tabular}{c|c|c|c|c}
\hline \hline $\begin{array}{c}\text { Speckle } \\
\text { Noise } \\
\text { Variance }\end{array}$ & $\begin{array}{c}\text { The } \\
\text { parameter } \\
\alpha\end{array}$ & $\begin{array}{c}\text { PSNR } \\
\text { with } \\
\text { the soft- } \\
\text { threshold } \\
\text { method }\end{array}$ & $\begin{array}{c}\text { PSNR } \\
\text { with } \\
\text { the hard- } \\
\text { threshold } \\
\text { method }\end{array}$ & $\begin{array}{c}\text { PSNR } \\
\text { with } \\
\text { the } \\
\text { proposed } \\
\text { method }\end{array}$ \\
\hline 0.010 & 0.3308 & 20.7587 & 20.9452 & 21.2978 \\
\hline 0.050 & 0.5903 & 20.2555 & 20.4035 & 20.7602 \\
\hline 0.300 & 0.8651 & 19.5840 & 19.6993 & 20.0564 \\
\hline 0.500 & 0.9034 & 19.7443 & 19.8224 & 20.1806 \\
\hline \hline
\end{tabular}

TABLE II. THE ESTIMATED COC, EPI, AND SSI OF AN ULTRASOUND IMAGE DENOISING USING THE PROPOSED METHOD AND OTHER METHODS.

\begin{tabular}{c|c|c|c|c|c|c}
\hline \multicolumn{1}{c|}{ Parameter } & \multicolumn{3}{|c|}{$\sigma=0.1$} & \multicolumn{2}{c}{$\sigma=0.5$} \\
\cline { 2 - 6 } Method & CoC & EPI & SSI & CoC & EPI & SSI \\
\hline Speckle noise image & 0.8351 & 0.4481 & 0.8630 & 0.6518 & 0.3656 \\
\hline The soft-threshold function & 0.9314 & 0.9277 & 0.9293 & 0.8325 & 0.8990 & 0.8316 \\
\hline The hard-threshold function & 0.8338 & 0.8057 & 0.8852 & 0.7958 & 0.8605 & 0.8188 \\
\hline Damodaran's method[9] & 0.9273 & 0.9155 & 0.9242 & 0.8589 & 0.8633 & 0.8043 \\
\hline Adaptive method[16] & 0.8488 & 0.8343 & 0.8816 & 0.7579 & 0.8546 & 0.7593 \\
\hline Our method with $\alpha=0.20$ & 0.9120 & 0.9261 & 0.9173 & 0.8964 & 0.8922 & 0.8486 \\
\hline Our method with $\alpha=0.50$ & 0.9314 & 0.9259 & 0.9293 & 0.9267 & 0.9024 & 0.8523 \\
\hline Our method with $\alpha=0.80$ & 0.9547 & 0.9242 & 0.9380 & 0.8565 & 0.8433 & 0.8370 \\
\hline \hline
\end{tabular}
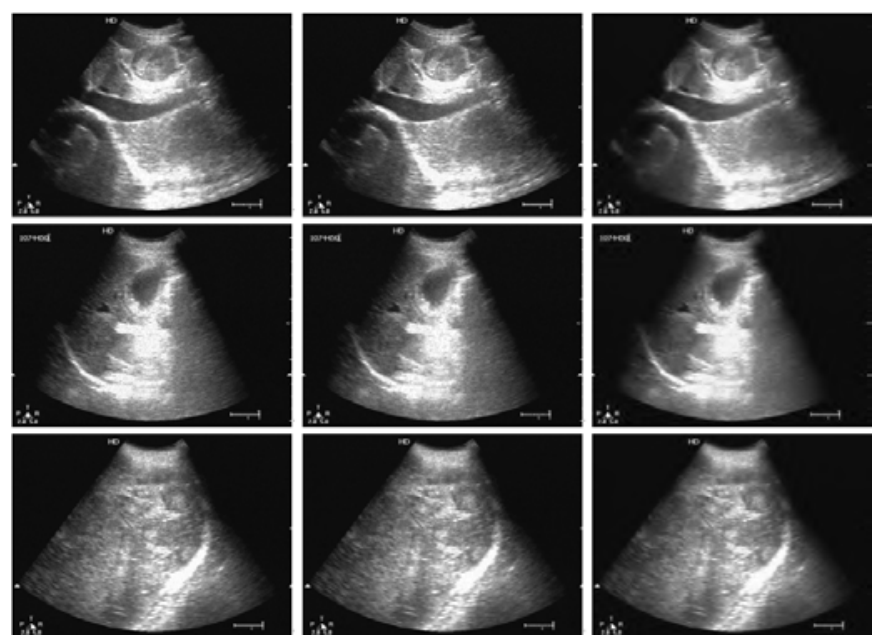

FIGURE II. DENOISING WITH DAMODARAN'S METHOD[9] AND THE PROPOSED METHOD FOR THREE LIVER IMAGES. FROM LEFT TO RIGHT: INPUT ULTRASOUND IMAGE, DENOISING WITH DAMODARAN'S METHOD [9] AND DENOISING WITH THE PROPOSED METHOD.

\section{SUMMARY}

In this work, we have investigated a hybrid threshold for ultrasound liver image denoising via the wavelet transform. We applied real medical ultrasound liver image to test the proposed method. Besides, a few metrics such as correlation coefficient, edge preservation index, and structural similarity index were estimated to evaluate the proposed method. The experimental results validate the wavelet-based hybrid thresholding technique for ultrasound liver image denoising.

\section{ACKNOWLEDGMENTS}

This work was supported in part by the Beijing University of Chemical Technology Interdisciplinary Funds for "Visual Media Computing" and Beijing Training Programme Foundation for the Talents No.2012B009016000004.

\section{REFERENCES}

[1] J.I. Koo, S.B. Park, Speckle reduction with edge preservation in medical ultrasonic images using a homogeneous region growing mean filter (HRGMF), Ultrasonic Imaging, 13(1991)211-237.

[2] B. Aiazzi, L. Alparone, S. Baronti, F. Lotti, Multi resolution local statistics speckle filtering based on a ratio Laplacian pyramid, IEEE Trans. Geosci. Remote Sense, 36(1998)1466-1476.

[3] A. Donka and M. Lyudmila. Contour segmentation in 2D ultrasound medical images with particle filtering, Machine vision and applications, 22(2012)551-561.

[4] H. Rabbani, M. Vafadust, P. Abolmaesumi, S.Gazor, Speckle Noise Reduction of Medical Ultrasound Images in Complex Wavelet Domain Using Mixture Priors, IEEE Transactions on Biomedical Engineering, 55(2008)2152-2160.

[5] K. Thangavel, R. Manavalan, I.Laurence Aroquiaraj, Removal of Speckle Noise from Ultrasound Medical Image based on Special Filters: Comparative Study, ICGST-GVIP Journal, 9(2009)25-32.

[6] M.I.H. Bhuiyan, M.O. Ahmad, M.N.S. Swamy, Spatially adaptive thresholding in wavelet domain for despeckling of ultrasound images, IET Image Processing, 3(2009)147-162. 
[7] A. Khare, M. Khare, Y. Jeong, H. Kim, M. Jeon, Despeckling of medical ultrasound images using Daubechies complex wavelet transform, Signal Processing, 90(2010)428-439.

[8] A.M.L. Lanzolla, G. Andria, F. Attivissimo, G.Cavone, N.Giaquinto. Improving B-mode ultrasound medical images. Proceedings of IEEE International Instrumentation and Measurement Technology Conference, 1(2011)1704-1708.

[9] N. Damodaran, S. Ramamurthy, S. Velusamy and G.K. Manickam. Speckle noise reduction in ultrasound biomedical B-scan images using discrete topological derivative, Ultrasound in medicine \& biology, 38(2012)276-286.

[10] J.M. Bioucas-Dias, M.A.T Figueiredo, Multiplicative noise removal using variable splitting and constrained optimization, IEEE Transactions on Image Processing, 19(2010)1720-1730.

[11] R. Sivakumar, M.K. Gayathri, D. Nedumaran, Speckle filtering of ultrasound B-scan images - A comparative study between spatial and diffusion filters, 2010 IEEE Conference on Open Systems, 1(2010)80-85.

[12] D.L. Donoho, I.M.Jonestone. Ideal spatial adaptation via wavelet shrinkage. Biometrika, 81(1994)425-455.

[13] A.G. Bruce, H.Y. Gao, Understanding WaveShrink: Variance and bias estimation. Biometrika, 83(1996)727-745.

[14] S.A. Harmoko, M.M. Marzuki, H. Aini, M. Oteh and N.F. Moha. Enhancement of myocardial boundary tracking using wavelet-based motion estimation, Journal of Information and Computational Science, 8(2011)1779-1792.

[15] H. Yu, L. Zhao, H. Wang, Image Denoising Using Trivariate Shrinkage Filter in the Wavelet Domain and Joint Bilateral Filter in the Spatial Domain, IEEE Trans. on Image Processing, 18(2009)2364 -2369.

[16] S. Gupta, R. Chauhan, S. Saxena. Homomorphic wavelet thresholding technique for denoising medical ultrasound images, Journal of Medical Engineering Technology, 29(2005)208-214. 